\title{
Assessing the incidence, epidemiological description and associated risk factors of breast cancer in western Amhara, Ethiopia
}

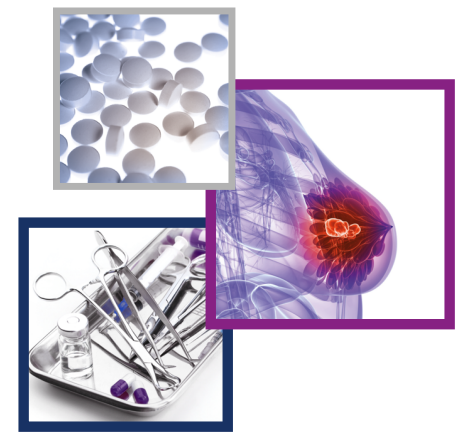

\author{
Lijalem M Tesfaw*,1 (iD), Teshome A Teshale² \& Essey K Muluneh ${ }^{1}$ (D) \\ ${ }^{1}$ Department of Statistics, Bahir Dar University, PO Box 79, Bahir Dar, Ethiopia \\ ${ }^{2}$ College of Medicine and Health Science, Tibebe Ghion Specialized Hospital, Bahir Dar University, Bahir Dar, Ethiopia \\ *Author for correspondence: lijalemmelie@gmail.com
}

\section{Practice points}

- Breast cancer is the most common cause of death that mostly occurs in middle- and low-income countries.

- Though it is prevalent disease in high- and low-income countries including Ethiopia, lack of awareness of the community about the disease, limited medical equipment are noticed.

- The treatment outcome of breast cancer patients depends on the residence of patients and, patients from rural area were less likely to improve.

- Compared with patients who received only chemotherapy treatment, those who received chemotherapy and surgery were more likely to improve if the patient capable to take it.

- As the stage of breast cancer increased prior to diagnosis, treatment outcome was less likely to improve even if appropriate treatment was provided.

- Since cancer disease is a noncommunicable disease and difficult to be cured after the disease test confirmed, it is advisable to take care of our body and prevent it. Subjects need to inspect their breast in different circumstances, for instance, during taking shower so that they can detect symptoms earlier. It helps to visit the hospital at an early stage when the cancer tumor is less invasive, did not spread to nearby parts of the particular body of the breast and where they would have more chance to get appropriate treatment and improve.

- Place of residence of patients has significantly associated with the incidence of breast and treatment outcome.

- Breast cancer is the most common malignancy disease for females ( $92.97 \%$ of the total) compared with males. It is started mainly between age 20 and 36 for both sexes.

- Type of treatment given to breasts cancer patients, place of residence, anemia complication and stage of the disease at diagnosis were independently associated with the patients' status after treatment.

Aim: This study aimed at assessing the incidence, epidemiological description and associated risk factors of breast cancer in western Amhara, Ethiopia. Patients \& methods: A total of 128 breast cancer patients being treated at Feleget Hiwot Referral Hospital (Bahir Dar, Ethiopia) were subjects of the study. Investigators controlled data quality and employed the ordinal logistic regression model to identify the effect of factors. Results: Of the 128 breast cancer patients under study, 119 (93\%) were females. Most of the patients, 67 (52.34\%), had advanced stage (IV) cancer that reduced their chance to recover after treatment. Conclusion: Patients who received chemotherapy and surgery for breast cancer were likely to improve than patients who received only chemotherapy. As the stage of breast cancer increased, the likelihood of the patient's status to be improved decreases. Patients from rural areas, where healthcare services are rarely available, were less likely to recover or improve as compared with those from urban areas.

First draft submitted: 8 May 2020; Accepted for publication: 22 July 2020; Published online: 21 August 2020

Keywords: breast cancer $\bullet$ epidemiology $\bullet$ incidence $\bullet$ oncology $\bullet$ ordinal logistic regression $\bullet$ treatment outcome

Noncommunicable disease such as cancer is the major cause of death globally. Cancer is a genetic disease that leads into unregulated growth and division of cells that form malignant tumor and invade the nearby parts of our body [1]. According to the Global Burden of Disease Cancer result report (2018), with death of 8 million, cancer is the second leading cause of death globally, next to cardiovascular disease. In women from high-, middle- and lowincome countries, breast cancer, which is characterized by uncontrolled growth of abnormal cells in the milk glands

Future Medicine 
of the breast or in the passages (ducts) that deliver milk to the nipples [2], is the most diagnosed type $(2,088,849$ cases, $12.2 \%$ of the total). It is the ninth most common cause of death in high-income countries and around $69 \%$ of all breast cancer deaths occur in middle- and low-income countries. In middle- and low-income countries, because of the absence of early recognition curricula, which leads to women attending with advanced-stage disease and a scarcity of satisfactory diagnosis and treatment capabilities, low-survival rates of women patients are common [3].

In women in both developed and developing countries, breast cancer has nowadays become one of the fastest growing illnesses. Many physical, chemical and genetic factors, including a high level of estrogen and progesterone pills, and late pregnancy (after 30 years), account for this. Despite the various ways developed for treating breast cancer, these methods treat cancer better in stages of I-II; cancer of stages III-IV are very serious and could hardly be treated effectively once they spread over the whole breast [2]. For women aged 15-49 years, twice as many breast cancer cases were recorded in developing countries rather than in developed countries. Factors of the variation include geography, lifestyle, environment, the presence of known risk factors, stage of disease at diagnosis and the availability of appropriate care [3].

Studies have reported that risk factors such as sex, obesity, lack of physical exercise, drinking alcohol, hormone therapy during menopause, ionizing radiation, age at first menstruation and old age affect the incidence of breast cancer $[4,5]$. Breast cancer could be reduced if appropriate treatment is provided for the patients and detection is made earlier but old age remains a risk factor for a poorer survival [5]. Besides the general ignorance of the public, lack of access to medical facilities, lack of more accurate diagnosis and economic circumstances cause the patients to come to the clinic at advanced stage of the cancer [6]. On the other hand, in developing countries like Ethiopia women will present themselves to a clinic at the advanced stage of breast cancer due to a limited number of hospitals.

Surgery and radiotherapy were reported to reduce the recurrence and death of breast cancer patients [7]. Though Feleget Hiwot Referral Hospital (FHRH; Bahir Dar, Ethiopia) has oncology ward for giving treatment to cancer patients, beginning from 2016, the hospital was unable to offer relevant treatment like radiotherapy. Patients to whom radiotherapy was prescribed are forced to go to Tikur Anbesa Hospital (Addis Ababa, Ethiopia), $564 \mathrm{~km}$ away from FHRH. As a result, although physicians write referrals to enable patients to get radiotherapy from Tikur Anbesa, patients do not go there for economic reasons, and lose the hope of cure, which can make patients vulnerable to death.

A study conducted in Tikur Anbesa Hospital reported that breast cancer accounts for 34\% of all female cancer cases, followed by cervical cancer at $16 \%$ [5]. Breast cancer frequently develops in cells lining milk ducts and the lobules that deliver the ducts with milk. There are more than 18 subtypes of breast cancer depending on cells in the breast affected [8]. This study does not take in to account the type of the breast cancer.

Globally, different studies detected many factors of breast cancer. In Africa, only a few studies investigated the incidence, epidemiological description and biological characteristics of breast cancer. In Ethiopia, in particular, the incidence, epidemiological description and associated risk factors of breast cancer received little attention. This study, therefore, was aimed to assess the incidence, epidemiological description of incidence and associated risk factors of breast cancer in western Amhara, Ethiopia. In Ethiopia, two limited prior studies explored the incidence, epidemiological description and associated risk factors of breast cancer. As a result, the findings of this study will be a useful input for stakeholder in terms of updating policy issues. For hospitals, it helps to provide appropriate prescriptions and treatments that matched with patient characteristics.

\section{Data \& methods}

Data description

The data used for this study were obtained from FHRH. Cancer patients at FHRH have their own oncology ward that assigns a unique patient ID and patients' characteristics were recorded on their registry cards based on their specified identification number. One hundred and thirty-nine breast cancer patients were recruited based on their identification number from oncology ward in the hospital. Patient registry cards that do not have all the required information of the patient, as well as patients who were not willing to participate in the study and patients who came from outside of western Amhara region of Ethiopia, were excluded. Finally, 128 breast cancer patients enrolled in the hospital from September 2016 to June 2019 were selected by experienced health professionals using a prespecified format designed by investigators and agreed to take part in the studies. Breast cancer patients enrolled from September 2016 were considered since the FHRH oncology ward was launched as of September 2016. The data were entered into the MS Excel and then exported to SAS 9.4 software to obtain the descriptive and inferential statistics. 


\begin{tabular}{|c|c|c|}
\hline Independent variables & & Frequency (\%) \\
\hline \multirow[t]{4}{*}{ Risk factors: } & $\begin{array}{l}\text { Gender } \\
0=\text { female } \\
1=\text { male }\end{array}$ & $\begin{array}{l}119(92.97) \\
9(7.03)\end{array}$ \\
\hline & $\begin{array}{l}\text { Blood type } \\
\text { A } \\
\text { B } \\
\text { AB } \\
\text { O }\end{array}$ & $\begin{array}{l}40(31.25) \\
34(26.56) \\
7(5.47) \\
47(36.22)\end{array}$ \\
\hline & $\begin{array}{l}\text { Residence } \\
\text { Urban } \\
\text { Rural }\end{array}$ & $\begin{array}{l}70(54.69) \\
58(45.31)\end{array}$ \\
\hline & Age & \\
\hline Prognostic factor: & $\begin{array}{l}\text { Stage } \\
\text { I } \\
\text { II } \\
\text { III } \\
\text { IV }\end{array}$ & $\begin{array}{l}6(4.69) \\
13(10.16) \\
42(32.81) \\
67(52.34)\end{array}$ \\
\hline Treatment: & $\begin{array}{l}\text { Treatment } \\
\text { Chemotherapy } \\
\text { Chemotherapy and surgery }\end{array}$ & $\begin{array}{l}59(46.09) \\
69(53.91)\end{array}$ \\
\hline \multicolumn{3}{|l|}{ Dependent variable } \\
\hline Treatment outcome: & $\begin{array}{l}\text { Treatment outcome } \\
1=\text { improved } \\
2=\text { same } \\
3=\text { deteriorate }\end{array}$ & $\begin{array}{l}43(33.60) \\
42(32.80) \\
43(33.60)\end{array}$ \\
\hline
\end{tabular}

\section{Variable description}

The description of independent and dependent variables considered in the study was revealed in Table 1 . The values of the variables obtained from patients' registry card were identified based on their unique patient ID. The independent variables, Table 1, were partitioned into four categories: risk factors, prognostic factors, complication and treatment based on the identities of the variable. Risk factors pertain to the factors associated with causing a breast cancer. These are determined by examining the factors influencing the incidence of new breast cancer cases such as age, gender, residence and blood type. Complication occurs when another disease arises as a result of breast cancer. Prognostic factors, on the other hand, are determined by following up the particular people who have breast cancer disease like stage of breast cancer. Using the classification system developed by the American Joint Committee on Cancer (IL, USA) that utilizes in part TNM scoring system, in which T, N and M refers to tumor size, nodal involvement and metastasis, respectively, in this study breast cancer was measured in four stages: I, II, III and IV, respectively, which is common for any other types of cancer too. The stages of breast cancer from I to IV refer to how much the cancer tumor is more advanced and is invading the near parts of the breast. A patient with stage IV cancer refers a cancer that was already very advanced during diagnosis. Most of the patients, 67 (52.34\%), present at hospital had advanced stage (IV) that reduces the likelihood of the patient to improve after treatment. Only six (4.69\%) patients took treatment at early stage of the cancer tumor.

The variable recorded as complication of breast cancer was anemia. While we realize that there may be factors that need to be checked as complicators of breast cancer, but there were not any recorded in the patients' registry card. The residence of each patients was recorded in terms of their district, in Table 2.

In FHRH, chemotherapy, surgery and palliative care are the most common treatments given for patients. Palliative care treatment is given for patients in cases where they do not have any ability to take chemotherapy as well as surgery. If the stage of cancer is low and respectable like stage I and II surgery is done before chemotherapy and if the stage is III or more chemotherapy is given alone. Doxorubicin, cyclophosphamide and methotrexate were typically used chemotherapy with other supportive medications like prechemotherapy rehydration and antiemetics. Nearly half $(46.09 \%)$ of the breast cancer patients took chemotherapy while the remaining patients took both chemotherapy and surgery. 
Table 2. Districts within zones and respective number of patients.

\begin{tabular}{|c|c|c|c|c|c|}
\hline \multirow[t]{2}{*}{ District } & \multicolumn{2}{|c|}{ Incidence } & \multirow[t]{2}{*}{ District } & \multicolumn{2}{|c|}{ Incidence } \\
\hline & Unstandardized (\%) & $\begin{array}{l}\text { Standardized } \\
\text { (per } 100,000)\end{array}$ & & Unstandardized (\%) & $\begin{array}{l}\text { Standardized } \\
\text { (per 100,000) }\end{array}$ \\
\hline Bahir Dar & $23(17.97)$ & 3.3729 & Dangla & $3(2.34)$ & 1.7452 \\
\hline Bahir Dar Zuria & $10(7.81)$ & 4.4684 & Fagita Lekoma & $4(3.13)$ & 2.9144 \\
\hline Dembecha & $5(3.91)$ & 3.5487 & Hulet ejun enese & $2(1.56)$ & 0.6626 \\
\hline Finote Selam & $2(1.56)$ & 6.8982 & Jawi & $2(1.56)$ & 2.3250 \\
\hline Goji Kolela & $3(2.34)$ & 2.5940 & Alefa & $4(3.13)$ & 2.2344 \\
\hline North Achefer & $3(2.34)$ & 1.5948 & Ankasha Guagusa & $8(6.25)$ & 3.6842 \\
\hline South Achefer & $2(1.56)$ & 1.1794 & Banja & $6(4.69)$ & 4.1494 \\
\hline Burie & $4(3.13)$ & 2.5577 & Goncha Sisoenese & $1(0.78)$ & 0.6206 \\
\hline Miecha & $4(3.13)$ & 1.2596 & Libo Kemkem & $4(3.13)$ & 11.8347 \\
\hline Merawi & $2(1.56)$ & 5.3593 & Debre Tabor & $4(3.13)$ & 6.3503 \\
\hline Mota & $4(3.13)$ & 7.3996 & Dera & $8(6.25)$ & 2.9905 \\
\hline Woberma & $2(1.56)$ & 1.8135 & Estie & $1(0.78)$ & 0.2786 \\
\hline Jabi Tehnan & $1(0.78)$ & 0.5154 & Ebinat & $2(1.56)$ & 0.8612 \\
\hline Yilmanadensa & $2(1.56)$ & 0.8561 & Farta & $1(0.78)$ & 0.3972 \\
\hline Bibugn & $2(1.56)$ & 2.2665 & Fogera & $2(1.56)$ & 0.8108 \\
\hline Debre Markos & $1(0.78)$ & 1.4017 & Gaynt & $2(1.56)$ & 0.5961 \\
\hline Belesa & $1(0.78)$ & 0.3958 & Smada & $1(0.78)$ & 0.4040 \\
\hline Enemay & $2(1.56)$ & 1.1117 & & & \\
\hline
\end{tabular}

Following medical diagnosis by the physician, treatment outcome/patient status will be identified and recorded into one of the five categories: cured, improved, same, deteriorate and death. As soon as the patient visited the hospital, the status will be recorded by the physician. After 3 months of follow-up of patients with treatment, the status of the patient was categorized as: Cured: if the patient was completely cured from cancer; Improved: if the patient has improvement status compared with the status while the patient visiting the hospital; Same: if there is no change noticed/observed on the patients after treatment; Deteriorate: if the patient getting worse after treatment; Died: if the patient passed away. It is primarily based on clinical assessment.

Of the total of 128 breast cancer patients under study $43(33.60 \%)$ patients improved after treatment. On the other hand, four $(3.13 \%), 39(30.47 \%)$ and $42(32.81 \%)$ of the patients died, deteriorated and did not show change of treatment outcome (remained the same), respectively, after the treatment. For the sake of simplicity in the analysis and interpretation of results, adjacent categories of the disease are merged and their status reclassified as improved (improved and cured), remained the same and deteriorated (deteriorated and death). From the total of 128 patients, $119(92.97 \%)$ were females and the rest nine $(7.03 \%)$ were males, Table 1.

\section{Ethics approval \& consent to participate}

Permission to undertake the study was obtained from both the ethical committees of Bahir Dar University and Felege Hiwot Referral Hospital. The researchers committed to the protection of the privacy of patients/participants medical information. Accordingly, identification of patients/participants was done only through numerical codes and collection of patients' medical information is made based on Ethiopian Ministry of health legislation to which FHRH is also committed.

\section{Statistical methods}

\section{Ordinal logistic regression modeling}

Ordinal logistic regression (OLR) is a type of analysis where the response variable has more than two categories having natural order or rank. Hence, ordered logit model is used to determine the association between the ordinal response variable and independent variables. OLR is a special case of generalized linear models in which the mean of the response variable is related to explanatory variables through a regression equation of logit link. The cumulative 


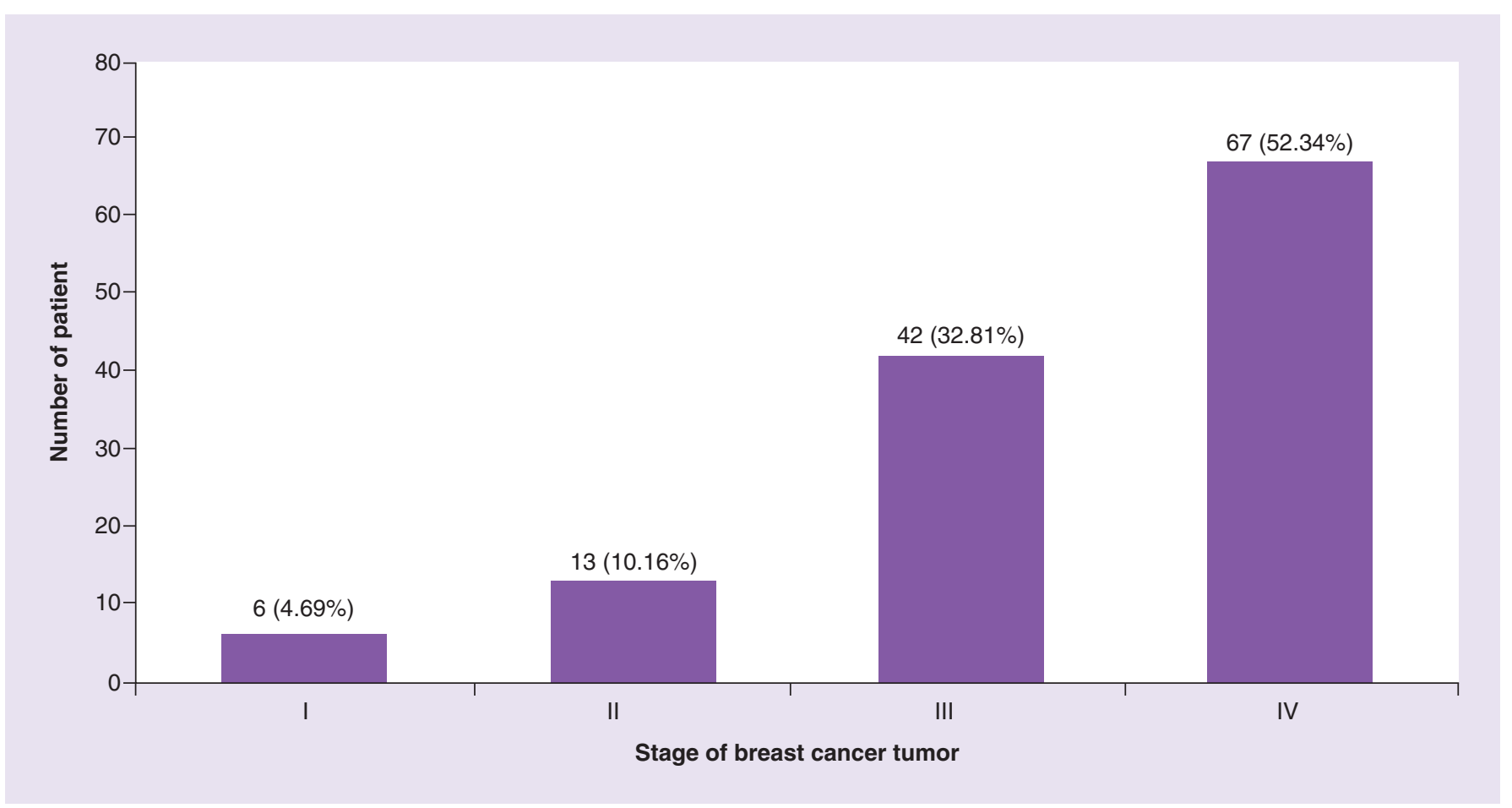

Figure 1. Distribution of patients within stages (I-IV) of breast cancer tumor.

logit model [9] is given in Equation 1 as follows:

$$
\begin{aligned}
\log i t(P[Y \leq j \mid x]) & =\log \left(\frac{P[Y \leq j \mid x]}{1-P[Y \leq j \mid x]}\right) \\
& =\alpha_{j}+\beta_{1} X_{1}+\beta_{2} X_{2}+\ldots+\beta_{p} X_{p}
\end{aligned}
$$

$\mathrm{j}=1,2, \ldots, \mathrm{J}$. where $\mathrm{J}$ is number of categories for the response variable $\mathrm{Y}$ and $X_{1}, X_{2}, \ldots, X_{p}$ are $\mathrm{p}$ independent variables while $i=1,2, \ldots, 128$ refers the $\mathrm{i}^{\text {th }}$ patient.

Like other forms of logistic regression models, ordinary logistic regression models are commonly interpreted using odds ratio (OR) [10], the ratio of two odds. The odds refers to the probability that treatment outcome $(\mathrm{Y})$ will fall in the $j^{\text {th }}$ or less categories, $\mathrm{P}[\mathrm{Y} \leq j]$ divided by its complement $\mathrm{P}[\mathrm{Y}>j]$. Defining the $\mathrm{X}$ as one of our covariates in the study, for two values $x_{1}$ and $x_{2}$ of $\mathrm{X}$, an OR comparing the cumulative probabilities is equal to:

$$
O R=\frac{O d d s(x 2)}{O d d s(x 1)}=\frac{P[Y \leq j \mid x=x 2] / P[Y>j \mid x=x 2]}{P[Y \leq j \mid x=x 1] / P[Y>j \mid x=x 1]}
$$

The basic idea underlying the assumption in OLR model is the idea of proportional odds/parallel lines. The proportional odds model considers that the cumulative logits can be represented as parallel linear functions of independent variables [9]. That is, for each cumulative logit the parameters of the models are identical, except for the intercept. Consequently, according to the proportional odds assumption, OR is the same for all categories of the response variable. Besides, to conduct OLR model, independent variables in the model need to be independent, in other words, there should be no multicollinearity. The Hosmer and Lemeshow test was used to check how well the model fits the data.

\section{Results}

Prior to fitting the statistical model, to identify the effect of independent variables on the treatment outcome, the incidence of breast cancer with independent factors was graphically explored. Patients stage significantly increased from stage I to stage IV during diagnosis (Figure 1), as was also shown in Table 1. The existence of patients with advanced-stage cancer weakens the likelihood of cure from treatment. As a result, from the 128 patients, only one 


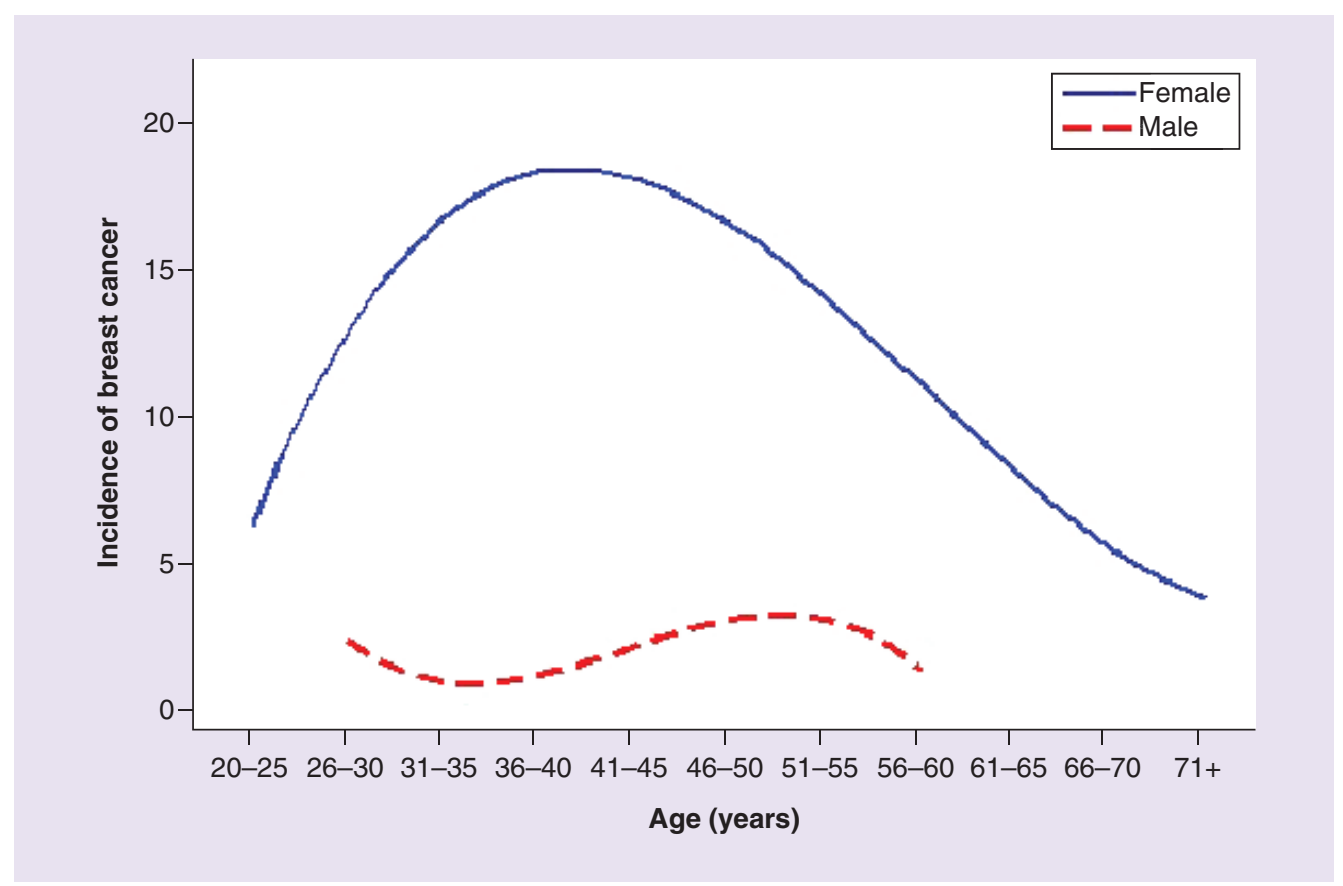

Figure 2. Distribution of incidence of breast cancer within each age group for females (blue color) and males (red color) using cubic estimated line.

patient was cured, and this has implications with respect to creating awareness about the decreased probability of cure.

For females, the incidence of breast cancer increased until 45 years of age, see Figure 2. For those older than 45 years, the incidence of breast cancer seems to decrease, indicating that breast cancer incidence was higher for women younger than 36 years. The incidence of breast cancer begins in the age 20-25 years for females and 26-30 years for males, because in this age, the size of breast for both sexes increases and become vulnerable to cancer. As illustrated in Table 1 and Figure 2, the incidence of breast cancer was very low for males than females. For males unlike for the females the incidence of breast cancer looks similar across all age groups. However, as could be seen in Figure 2, the data each age group did not consider the size of populations at risk. Thus, the higher incidence of breast cancer among females in the age 35-50 years may be due to the high number of females belonging to this age group.

The patients in this study were obtained from 35 districts in west Amhara region based on the evidence from patients' registry card in oncology ward of FHRH. The incidence of breast cancer patients in each district displayed in Table 2 includes both unstandardized (\%) and standardized (per 100,000) incidences.

Unstandardized (\%) incidence of breast cancer patient in each district in Table 2 reflects the number of breast cancer patients in each district regardless of the size of the population at risk within the districts and corresponding proportion out of the total breast cancer patients (in brackets). The unstandardized incidence of breast cancer was maximum at Bahir Dar district 23 (17.97\%) while minimum incidence was observed at Debre Markos, Belesa and Jabi Tehnan districts $1(0.78 \%)$. However, the unstandardized estimate of incidence of breast cancer presented in Table 2 does not take into account the total number of populations at risk in each district. The highest incidence of breast cancer in Bahir Dar district might be because of high number of inhabitants/populations at risk; lowest incidence of breast cancer in Debre Markos, Belesa and Jabi Tehnan districts might be because of minimum number of inhabitants/populations at risk. As a result, the incidence for two districts may appear different, but this difference may be due entirely to the different number of population at risk distributions within the district, rather than to a difference in the underlying incidence of breast cancer disease. Hence, to make comparable incidence of breast cancer in each district the population at risk was standardized per 100,000 population. Therefore, the highest incidence of breast cancer was recorded at Libo Kemkem (11.8347) and the lowest was from Estie district (0.2786) 


\begin{tabular}{|c|c|c|c|c|c|}
\hline \multirow[t]{2}{*}{ Covariates } & \multirow[t]{2}{*}{ Level } & \multicolumn{3}{|c|}{ Treatment outcome } & \multirow[t]{2}{*}{ p-value } \\
\hline & & Improved $\mathrm{n}(\%)$ & Same n (\%) & Deteriorate $\mathrm{n}(\%)$ & \\
\hline Treatment & $\begin{array}{l}\text { Chemotherapy and } \\
\text { surgery }\end{array}$ & $33(76.7)$ & $18(42.9)$ & $18(41.9)$ & 0.001 \\
\hline \multirow[t]{2}{*}{ Blood group } & A & $15(34.9)$ & $11(26.2)$ & $14(32.6)$ & \multirow[t]{2}{*}{0.921} \\
\hline & 0 & $16(37.2)$ & $17(40.5)$ & $14(32.6)$ & \\
\hline \multirow[t]{2}{*}{ Residence } & Urban & $22(64.71)$ & $65(63.64)$ & $21(44.68)$ & \multirow[t]{2}{*}{0.032} \\
\hline & Rural & $12(35.29)$ & $20(36.36)$ & $26(55.32)$ & \\
\hline \multirow[t]{2}{*}{ Sex } & Female & $40(93.0)$ & $39(92.9)$ & $40(93.0)$ & \multirow[t]{2}{*}{0.999} \\
\hline & Male & $3(7.0)$ & $3(7.1)$ & $3(7.0)$ & \\
\hline \multirow{3}{*}{ Stage } & II & $8(18.6)$ & $3(7.1)$ & $2(4.7)$ & \multirow{3}{*}{$<0.001$} \\
\hline & IIII & $26(60.5)$ & $11(26.2)$ & $5(11.6)$ & \\
\hline & IV & $5(11.6)$ & $27(64.3)$ & $35(81.4)$ & \\
\hline
\end{tabular}

Bold p-value (less than 0.05 ) indicates that variables are significantly associated with treatment outcome.

\begin{tabular}{|c|c|c|c|c|c|c|}
\hline \multirow[t]{2}{*}{ Treatment } & & \multicolumn{4}{|c|}{ Stage } & \multirow[t]{2}{*}{ p-value } \\
\hline & & I & II & III & IV & \\
\hline Chemotherapy & $\mathrm{n}(\%)$ & $3(50.0)$ & $6(46.2)$ & $9(21.4)$ & $41(61.2)$ & 0.001 \\
\hline $\begin{array}{l}\text { Chemotherapy and } \\
\text { surgery }\end{array}$ & $\mathrm{n}(\%)$ & $3(50.0)$ & $7(53.8)$ & $33(78.6)$ & $26(38.8)$ & \\
\hline
\end{tabular}

More than three quarters $(76.7 \%)$ of the recovered patients took chemotherapy and surgery. As shown in Table 3, patients who did not show change of treatment outcome and those who deteriorated were 42.9 and $41.9 \%$, respectively. This indicates that chemotherapy and surgery treatments are advisable if the patients do not interrupt the treatment. A significant association is seen between treatment given to patients and their treatment outcome $(\mathrm{p}=0.001<0.05)$. Variables with bold $\mathrm{p}$-value (such as anemia, place of residence, treatment and stage) indicate the variable is independently associated with treatment outcome at $5 \%$ level of significance. However, it does not show the joint influence of many variables on the treatment outcome. For this reason, statistical modeling involving all the variables under consideration was demonstrated in Table 7 . For the given data where the response variable, treatment outcome, has categorical values which can be put in natural order (improved, same and deteriorate), OLR model is the right statistical model. The model parameters indicate the degree of influence that variable has on the patient status.

Patients who have anemia complication during diagnosis were less likely to improve than those who were not anemic. Stage of breast cancer tumor during diagnosis has its own association with treatment outcome $(\mathrm{p}<0.001)$. Among patients whose status was a deteriorate 35 (81.4\%) had stage IV breast cancer tumor. Among the stage III, most of the patients $27(64.3 \%)$ did not have change of treatment outcome after treatment. Patients who had a stage IV breast cancer tumor during diagnosis were more likely to have a deteriorate treatment outcome. Patients with early stage of cancer have more chance of a better patient status after treatment.

Table 4 shows that the association between stage of breast cancer tumor and treatment prescribed to the patient was significant, p-value $(0.001<0.05)$.

In Table 3, the effect of each independent variable was checked in the bivariate analysis. However, to know the overall effect of a variable the effect of the other variable should be considered. In fact, considering all variables in our model has its own effect because some of the variables that enable us to know the effect of other covariates may not be necessary. Hence, variable selection using stepwise method was done by $20 \%$ entry and $25 \%$ stay level 
Table 5. Model adequacy checking.

\begin{tabular}{|l|lll} 
& Proportional odds & \multicolumn{2}{c}{ Hosmer and Lemeshow test } \\
\cline { 1 - 4 }$\chi^{2}$ & p-value & $\chi^{2}$ & p-value \\
\hline 7.89 & 0.25 & 26.15 & 0.08 \\
\hline
\end{tabular}

Table 6. Association of predicted probabilities and observed treatment outcome.

Concordant Discordant

of significance [10]. All possible pairwise interaction between variables was checked and none of the interaction effects were significant. Finally, OLR model was fitted after the model's assumption such as proportional odds and multicollinearity were checked.

\section{Model adequacy checking}

Before fitting the OLR model, we need to ensure the adequacy of the model. The pillar assumption of OLR, proportional odds, was checked in which the $\chi^{2}$ statistic equal $8.86(\mathrm{p}=0.26>0.05)$, indicating that the proportional odds assumption for the cumulative logit of our data was valid (see Table 6). The goodness of fit of the data was checked using the Hosmer and Lemeshow test [10]. The value of the $\chi^{2}$ statistics was 26.15 $(p=0.08>0.05)$, indicating again that the model is a good fit to the data, see Table 5 . To handle multicollinearity problem, some of the independent variables were removed using stepwise method of variable selection and it was noted that no multicollinearity problem was noticed in the data.

Having checked the goodness of fit of the model, how good the model is in predicting the data were evaluated using values of concordant and discordant, as presented in Table 6. Concordant value of $78.1 \%$ shows that as many as $78.1 \%$ of the patients with treatment outcome of $(<\mathrm{j})$ have better chance in predicting the category $(<\mathrm{j})$. A discordant value of $20.9 \%$ indicates that as many as $20.9 \%$ patients with treatment outcome $(>\mathrm{j})$ have a better chance of predicting the category $(<\mathrm{j})$. This indicates that the model's adequacy in reflecting the association was very good.

\section{Parameter estimates}

Based on the results revealed in Table 7, the final estimated OLR model is given by:

$$
\begin{gathered}
\log i t\left(P\left[Y_{i} \leq j\right]\right)=\alpha_{j}+0.1892 \text { Anemia }_{i}-0.6801 \text { Residence }_{i}-0.4358 \text { Chemotheraphy }_{i}+ \\
\\
0.7199 \text { Stage }_{i}+0.6421 \text { StageII }_{i}+0.4155 \text { StageII }_{i}
\end{gathered}
$$

Positive estimated coefficients show that the treatment outcome was more likely to fall at the high end of the scale as the corresponding variable $\mathrm{X}$ increases and vice versa for of the quantitative variables. On the other hand, for qualitative variables positive estimated coefficients refer to treatment outcomes that are more likely to fall at the lower end of the scale for the dummy category compared with the reference category. The OR estimates are used to compare the odds of the patient to fall in the $\mathrm{j}^{\text {th }}$ or lower treatment outcome between two categories of the factor. OR simply can be calculated by exponentiating the estimated coefficients of the corresponding variables, $e^{\beta} i$. The fitted model in Table 7 indicates that treatment and stage of breast cancer tumor at diagnosis have significant effect (p-value in bold $<0.05$ ) on log odds of treatment outcome to be in category j or below. The ordered logit of patients who received chemotherapy in a lower treatment outcome category was 0.4358 -times lower than patients who received chemotherapy and surgery. This indicates that the estimated odds of patients who received chemotherapy was 0.4320 -times the estimated odds of patients who received chemotherapy and surgery, which indicates that, keeping other variables constant, the estimated odds of patients who fall in the lower direction of treatment outcome category was lower by $56.80 \%$ of estimated odds of patients who received chemotherapy and surgery. Therefore, patients who received chemotherapy were less likely to better position of treatment outcome than patients who received chemotherapy and surgery. The ordered logit of patients who had stage I, stage II and stage III in a lower treatment outcome category (better chance to improve or cure) were $0.7199,0.6421$ and 0.4155 -times higher than patients who had stage IV. Hence, the estimated odds of patients who had stage I, stage II and stage III breast 


\begin{tabular}{|c|c|c|c|c|c|c|c|c|}
\hline \multirow[t]{2}{*}{ Parameter } & \multirow[t]{2}{*}{ Estimate } & \multirow[t]{2}{*}{ St error } & \multirow[t]{2}{*}{ p-value } & \multicolumn{5}{|c|}{ Odds ratio estimates } \\
\hline & & & & Effect & Point & Estimate & Interval & Estimate \\
\hline \multicolumn{9}{|l|}{ Intercept } \\
\hline Improved & -0.4055 & 0.02979 & & & & & & \\
\hline Same & 1.5914 & 0.3414 & & & & & & \\
\hline Age & 1.1241 & 1.3582 & 0.3221 & & 1.223 & & 0.689 & 1.345 \\
\hline Anemia & & & 0.1772 & & & & & \\
\hline No & 0.1892 & 0.1893 & & No vs Yes & 1.667 & & 0.794 & 3.501 \\
\hline Yes $^{\dagger}$ & 0.0000 & & & & & & & \\
\hline Residence & & & 0.036 & & & & & \\
\hline Rural & -0.6801 & 0.1025 & & Rural vs urban & 0.5066 & & 0.2370 & 0.8501 \\
\hline Urban $^{\dagger}$ & 0.0000 & & & & & & & \\
\hline Treatment & & & 0.0290 & & & & & \\
\hline Chemotherapy & -0.4358 & 0.192 & & Chemo vs comb & 0.432 & & 0.204 & 0.918 \\
\hline Chemotherapy and surgery ${ }^{\dagger}$ & 0.0000 & & & & & & & \\
\hline Stage & & & 0.0001 & & & & & \\
\hline 1 & 0.7199 & 0.6513 & & I vs IV & 12.230 & & 2.104 & 71.105 \\
\hline II & 0.6421 & 0.4856 & & II vs IV & 9.1770 & & 2.551 & 33.010 \\
\hline III & 0.4155 & 0.3663 & & III vs IV & 11.323 & & 4.536 & 28.264 \\
\hline $\mathrm{IV}^{\dagger}$ & 0.0000 & & & & & & & \\
\hline
\end{tabular}

cancer tumor to be in the better patient status or treatment outcome is equal to $12.230,9.1770$ and 11.323-times the estimated odds of patients who had stage IV breast cancer. This indicates that patients who had stage I, stage II and stage III breast cancer were more likely to fall at the lower category of treatment outcome than patients who had had stage IV breast cancer. Place of residence of patients had significant effect on patients' treatment outcome $(p=0.036<0.05)$. The estimated odds of patients from rural area to be in the lower category of patient status was equal to 0.5066-times the estimated odds of patients from urban area. This indicates that patients from rural were less likely fall at the lower category of treatment outcome than patients from urban. It was also identified that anemia is not a significant complication following disclosure of patients of breast cancer.

\section{Discussion}

This research paper, based on the hospital records of breast cancer patients at the oncology ward of FHRH, depicted the prevalence of breast cancer in the districts of western Amhara region, Ethiopia, as well as the effect of common factor's on treatment outcome. Type of treatment given to breast cancer patients, place of residence, anemia complication and stage of the disease at diagnosis were independently associated with the treatment outcome or patients' status after treatment. Breast cancer mainly started from age 20 and 36 for females and males, respectively. Breast cancer was most common malignancy disease for females ( $92.97 \%$ of the total) compared with males, which is consistent with studies elsewhere [2,3]. However, no significant breast cancer treatment outcome difference was noticed between males and females. Unadjusted age rates of incidence of breast cancer in females increased till age 45. High incidence of breast cancer was recorded for the age group 36 to 45. Most of the patients visited hospital with advanced stage (stage IV $52.34 \%$ of the total) of the breast cancer tumor and only, $4.69 \%$ of patients who visited the hospital had stage I cancer. This is mainly because the country has inadequate oncology services established in some main cities and a few doctors and nurses struggling to serve a nation with more than 100 million people and with lifestyle changes that expose people to cancer. This has become a cause of death for cancer patients in the area. In fact, as stated in [3], in middle- and low-income countries are explained mainly by lack of early detection of programs resulting in high proportion of women with advanced stage of cancer disease owing to the lack of adequate diagnosis and treatment. Hence, patients who had advanced stage of breast cancer tumor were less likely improve relative to patients who had low-stage breast cancer. In contrast of studies in [11,12], in this study insignificant treatment outcome difference between patients' age. 
It is noted that for breast cancer patients, in addition to chemotherapy, using surgery and radiotherapy is likely to reduce the recurrence and death of patient [8], which is in line with our findings as compared with patients who received only chemotherapy, giving both chemotherapy and surgery have more chance of improving for breast cancer treatment outcome. Studies in [13] and [14] demonstrated that geographic or spatial epidemiology offers insight into ways that individual characteristics, community attributes and physical environments interact to produce distinctive risk, illness and disease management patterns. This finding supported in this study, which reported that significant treatment outcome differences between patients who were living in rural and urban noticed.

\section{Conclusion}

In the western Amhara region of Ethiopia, the treatment outcome of breast cancer patients depends on the residence of patients and, hence, patients from rural villages were less likely to improve. Compared with patients who received only chemotherapy treatment, those who received chemotherapy and surgery were more likely to improve. As the stage of breast cancer increased prior to diagnosis, treatment outcome was less likely to improve even if appropriate treatment was provided. It is noted that any women need inspect their breast in different circumstances, as for instance, during taking shower so that they can detect symptoms earlier, and visit hospital at an early stage when the cancer tumor is less invasive, did not spread to nearby parts of the particular body of the breast, and where they would have more chance to get relevant treatment and improve. It is further noted that the prescription of treatment for patients depend on the stage of breast cancer the patient had.

Author contributions

LM Tesfaw had the idea of the research question for the study and proposed the first draft. LM Tesfaw conducted data analysis, interpretation and prepared all figures and tables. TA Teshale collected data with LM Tesfaw and edited the manuscript. EK Muluneh edited and revised the manuscript. Finally, all the authors discussed the results and contributed to the final manuscript.

Acknowledgments

The authors thank personnel at Bahir Dar Felege Hiwot Referral Hospital who helped us to collect the data and expert assistance.

Financial \& competing interests disclosure

The authors have no relevant affiliations or financial involvement with any organization or entity with a financial interest in or financial conflict with the subject matter or materials discussed in the manuscript. This includes employment, consultancies, honoraria, stock ownership or options, expert testimony, grants or patents received or pending, or royalties.

The manuscript was edited for language by Berhanu Engdaw (PhD), Department of English, Bahir Dar University.

Ethical conduct of research

Permission to undertake the study was obtained from both the ethical committees of Bahir Dar University and Felege Hiwot Referral Hospital. The researchers committed to protection of the privacy of patients/participants medical information. Accordingly, identification of patients/participants was done only through numerical codes and collection of patients' medical information is made based on Ethiopian Ministry of health legislation to which FHRH is also committed.

Availability of data \& material

The datasets for generated analyses during the study are available at Bahir Dar Felege Hiwot Referral Hospital data repository.

Open access

This work is licensed under the Attribution-NonCommercial-NoDerivatives 4.0 Unported License. To view a copy of this license, visit http://creativecommons.org/licenses/by-nc-nd/4.0/

\section{References}

Papers of special note have been highlighted as: $\bullet$ of interest; $\bullet \bullet$ of considerable interest

1. Bray F, Ferlay J, Soerjomataram I et al. Global cancer statistics 2018: GLOBOCAN estimates of incidence and mortality worldwide for 36 cancers in 185 countries. CA Cancer J. Clin. 68(6), 394-424 (2018).

2. Bray F, Ferlay J, Soerjomataram I, Siegel RL, Torre LA, Jemal A. Global cancer statistics 2018: GLOBOCAN estimates of incidence and mortality worldwide for 36 cancers in 185 countries. CA Cancer J. Clin. 68(6), 394-424 (2018).

-. An excellent paper that assesses the prevalence and distribution of cancer worldwide.

3. Saboula N, El-Sayed SM. Effectiveness of application of PLISSIT counseling model on sexuality for breast cancer's women undergoing treatment. Am. J. Nurs. Sci. 4(4), 218-230 (2015). 
4. Gøtzsche PC, Jørgensen KJ. Screening for breast cancer with mammography. Cochrane Database Syst. Rev. 12(6), CD001877 (2009).

- A very good paper explaining how breast cancer is screened by mammography.

5. Hadgu E, Seifu D, Tigneh W et al. Breast cancer in Ethiopia: evidence for geographic difference in the distribution of molecular subtypes in Africa. BMC Womens Health. 18(1), 1-8 (2018).

6. Singh G, Singh DP, Gupta D, Muralikrishna BV. Neoadjuvant chemotherapy in locally advanced breast cancer. J. Surg. Oncol. 61(1), 38-41 (2010).

7. Haagensen CD, Stout AP. Carcinoma of the breast. II - criteria of operability. Ann. Surg. 118(6), 1032-1051 (2016).

8. Kabel AM, Baali FH. Breast cancer: insights into risk factors, pathogenesis, diagnosis, and management. J. Cancer Res. Treat. 3(2), 28-33 (2015).

-• Brief explanation of associated risk factors of breast cancer and gives insight to which has an effect.

9. Hosmer DW, Lemeshow S, Sturdivant RX. Applied Logistic Regression. John Wiley and Sons, NJ, USA (2007).

10. Hosmer DW Jr, Lemeshow S, Sturdivant RX. Applied Logistic Regression, 3rd Edition. John Wiley and Sons, NJ, USA (2013).

11. Anders CK, Johnson R, Litton J, Phillips M, Bleyer A. Breast cancer before age 40 years. SF J. Med. Oncol. Cancer. 36(3), 237-249 (2009).

12. Kroenke CH, Rosner B, Chen WY, Kawachi I, Colditz GA, Holmes MD. Functional impact of breast cancer by age at diagnosis. J. Clin. Oncol. 22(10), 1849-1856 (2004).

13. Best N, Richardson S, Thomson A. A comparison of Bayesian spatial models for disease mapping. Stat. Methods Med. Res. 14(1), 35-59 (2005).

14. Gregorio DI, Cromley E, Mrozinski R, Walsh SJ. Subject loss in spatial analysis of breast cancer. Health Place 5(2), $173-177$ (2001).

- Justifies the spatial analysis of breast cancer and whether breast cancer differ based on geography. 
